\title{
Copper catalyzed synthesis of quinolinopyrano[2,3-b]carbazole derivatives via Povarov reaction
}

\author{
SUBBURETHINAM RAMESH and RAJAGOPAL NAGARAJAN* \\ School of Chemistry, University of Hyderabad, Hyderabad 500 046, India \\ e-mail:rnsc@uohyd.ernet.in; naga_indole@yahoo.co.in
}

MS received 6 January 2014; revised 9 January 2014; accepted 12 February 2014

\begin{abstract}
An efficient procedure for the synthesis of pyranocarbazole derivatives using copper catalysed Povarov reaction between aromatic amines and $O$-propargyl carbazole carboxaldehyde has been developed. Ionic liquid is found to be a good solvent medium.
\end{abstract}

Keywords. Povarov reaction; copper(I)iodide; ionic liquid; pyranocarbazole.

\section{Introduction}

Carbazoles represent an important family of heterocyclic compounds that are present in many natural products. ${ }^{1}$ Naturally occurring carbazoles and synthetic carbazole analogues are known to exhibit promising biological applications. ${ }^{1}$ Among these, pyranocarbazole alkaloids have spared in the natural product synthesis due to their wide isolation from various plants. ${ }^{1,2}$ Some of the important pyranocarbazole alkaloids are shown in figure 1.

Pyranocarbazole analogues showed antioxidant activity at $33.1 \mu \mathrm{g} / \mathrm{mL},{ }^{3 a}$ antiosteoporotic activity in the CAT-B mode ${ }^{3 \mathrm{~b}}$ and cytotoxicity against leukemia and colon cancer cell lines. ${ }^{3 \mathrm{c}}$ Usually, an oxidative cyclization of a hydroxy prenyl or hydroxy geranylcarbazole has been used for the construction of pyran ring present in this class of alkaloids. 2-Hydroxy-3-methylcarbazole is recognized as an efficient reaction precursor for the synthesis of Mahanimbine ${ }^{4 a}$ and Girinimbine. ${ }^{4 b}$ Knölker et al. utilised palladium catalysed BuchwaldHartwig amination followed by double aromatic $\mathrm{C}-\mathrm{H}$ bond activation of biaryl compound. ${ }^{4 c}$ The same group reported tandem $\mathrm{C}-\mathrm{C}$ and $\mathrm{C}-\mathrm{N}$ bond formation using aromatic substitution of aminochromene with iron carbonyl complex followed by $\mathrm{I}_{2}$ mediated oxidative coupling of biaryl compound. ${ }^{4 \mathrm{~d}}$ Lebold et al. demonstrated the synthesis of pyrano[2,3-c]carbazole alkaloids (Eustifoline-A and Eustifoline-B) using a Diels-Alder reaction of quinone imine followed by Plieninger indolization reaction. ${ }^{4 \mathrm{e}}$ Recently, our group utilized Larock's iodocyclization of $O$-propargylated

\footnotetext{
*For correspondence
}

carbazoles for the synthesis of pyrano[3,2- $b]$ and pyrano[2,3-c] carbazole derivatives. ${ }^{4 \mathrm{f}}$

Chromenes and chromenoquinolines are an important class of heterocyclic compounds which have attracted considerable attention, due to their diverse biological activities ${ }^{5}$ and hence many synthetic strategies have been developed by various groups. ${ }^{6}$ We anticipated as in figure 2 that the presence of both pyranocarbazole and chromenoquinoline units in a single molecule could make more biological applications. Povarov reaction has been recognized as a practical method to construct quinoline or tetrahydroquinoline compounds. ${ }^{7}$ We envisioned that such a strategy if applied to an appropriate precursor could make interesting synthetic application for the pyranocarbazole derivatives. Though there are many synthetic approaches for making pyrano[3,2-a], [2,3$c]$ and $[3,2-b]$ carbazoles, ${ }^{4}$ synthesis of pyrano[2,3$b$ carbazole derivatives have been scarcely studied. Based on the important biological profile of pyranocarbazoles and our continuing interest in Povarov reaction, ${ }^{\text {6e-g }}$ we report here Povarov reaction between $O$-propargyl carbazole carboxaldehyde and anilines to give quinolinopyrano[2,3- $b]$ carbazole derivatives.

\section{Experimental}

\subsection{General information}

The melting point of the products was recorded on a Superfit (India) capillary melting point apparatus and is uncorrected. Infrared spectra were recorded on a JASCO FT/IR-5300 spectrophotometer. All the spectra were calibrated against polystyrene absorption at 

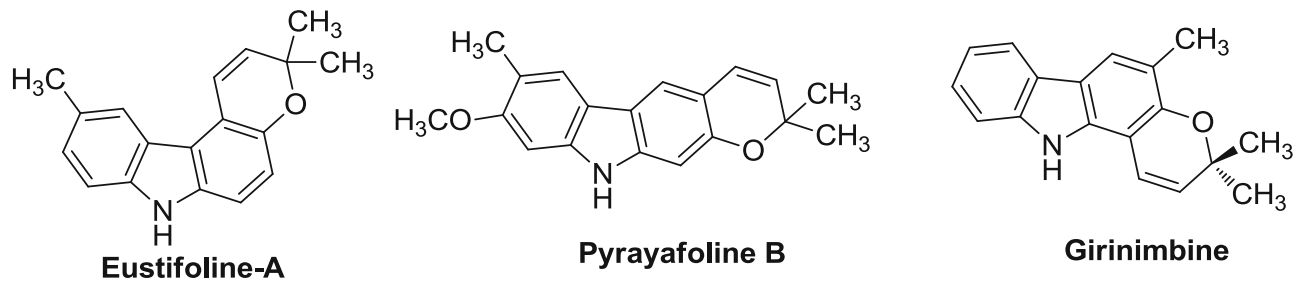

Figure 1. Some of the important pyranocarbazole alkaloids.

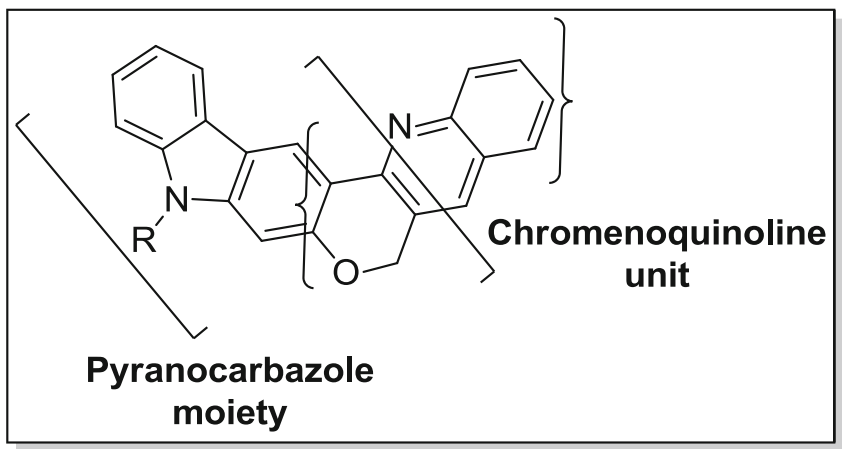

Figure 2. Quinolinopyranocarbazole.

$1601 \mathrm{~cm}^{-1}$. Solid samples were recorded as KBr wafers and liquid samples as thin film between $\mathrm{NaCl}$ plates or solution spectra in DCM. ${ }^{1} \mathrm{H}$ NMR and ${ }^{13} \mathrm{C}$ NMR spectra were recorded on BRUKER-AVANCE-400/500 spectrometers. ${ }^{1} \mathrm{H}$ NMR (400 or $500 \mathrm{MHz}$ ) spectra for all the samples were measured in chloroform- $d$, unless otherwise mentioned ( $\delta=2.50 \mathrm{ppm}$ for ${ }^{1} \mathrm{H}$ NMR in the case of DMSO- $\left.d_{6}\right)$, with TMS $(\delta=0 \mathrm{ppm})$ as an internal standard. ${ }^{13} \mathrm{C}$ NMR $(100$ or $125 \mathrm{MHz})$ spectra for all the samples were measured in chloroform- $d$, unless otherwise mentioned (in the case of DMSO- $d_{6}$, $\delta=39.70 \mathrm{ppm}$ its middle peak of the septet), with its middle peak of the triplet ( $\delta=77.10 \mathrm{ppm}$ ) as an internal standard. Mass spectral data were obtained from HRMS (ESI). The TOF and quadrupole mass analyzer types are used for the HRMS measurements. All the cases DCM or $\mathrm{MeOH}$ were used to dissolve the compounds.

2.1a Procedure for the preparation of 2-hydroxy-9methyl-9H-carbazole-3-carbaldehyde (2): The reaction mixture of aldehyde (1) $(1.0 \mathrm{mmol})$ and pyridine $\mathrm{HCl}$ salt (10 equiv.) was heated under microwave condition for $10 \mathrm{~min}$. The crude was extracted using dichloromethane and evaporated under reduced pressure. Pure product was obtained as a colourless solid through silica gel column chromatography using 3\% ethyl acetate in hexanes as eluent; Yield: 63\%; m.p. 165-167 ${ }^{\circ} \mathrm{C}$; IR (KBr): 3357, 1936, 1147, $665 \mathrm{~cm}^{-1} ;{ }^{1} \mathrm{H}$
NMR (400 MHz, $\mathrm{CDCl}_{3}$, TMS) $\delta: 11.53(1 \mathrm{H}, \mathrm{s}), 9.92$ $(1 \mathrm{H}, \mathrm{s}), 8.13(1 \mathrm{H}, \mathrm{s}), 7.99(1 \mathrm{H}, \mathrm{d}, J=7.6 \mathrm{~Hz}), 7.47$ $(1 \mathrm{H}, \mathrm{t}, J=7.2 \mathrm{~Hz}), 7.36(1 \mathrm{H}, \mathrm{d}, J=8.4 \mathrm{~Hz}), 7.29(1 \mathrm{H}$, $\mathrm{t}, J=7.6 \mathrm{~Hz}), 6.79(1 \mathrm{H}, \mathrm{s}), 3.77(3 \mathrm{H}, \mathrm{s}) ;{ }^{13} \mathrm{C} \mathrm{NMR}$ $\left(100 \mathrm{MHz}, \mathrm{CDCl}_{3}, \mathrm{TMS}\right) \delta: 195.0,161.3,146.9,142.1$, 127.1, 126.0, 123.1, 120.7, 119.7, 117.2, 115.0, 108.9, 94.9 (Aromatic C); 29.4 (Aliphatic C); HRMS (ESIMS) calcd. for $\mathrm{C}_{14} \mathrm{H}_{11} \mathrm{NO}_{2}(\mathrm{M}+\mathrm{H}) ; 226.0868$, found: 226.0865

2.1b Procedure for the preparation of 9-methyl-2(prop-2-yn-1-yloxy)-9H-carbazole-3-carbaldehyde (3): To the reaction mixture of 2-hydroxy-9-methyl-9 $\mathrm{H}$ carbazole-3-carbaldehyde (2) $(1 \mathrm{mmol})$ and $\mathrm{K}_{2} \mathrm{CO}_{3}$ ( 5 equiv.) in acetone was added propargyl bromide $(1.5 \mathrm{mmol})$. Then the reaction mixture was heated at reflux temperature of acetone. After completion of the reaction as indicated by TLC, the solvent was evaporated and extracted with ethylacetate. The solvent was removed under reduced pressure and pure product was obtained through silica gel column chromatography with $2 \%$ ethyl acetate in hexanes. m.p. $124-126^{\circ} \mathrm{C}$; IR (KBr): 3457, 2829, 1697, 1147, $645 \mathrm{~cm}^{-1}$; ${ }^{1} \mathrm{H}$ NMR (400 MHz, $\left.\mathrm{CDCl}_{3}, \mathrm{TMS}\right) \delta: 10.49(1 \mathrm{H}, \mathrm{s}), 8.65(1 \mathrm{H}, \mathrm{s})$, $8.01(1 \mathrm{H}, \mathrm{d}, J=7.6 \mathrm{~Hz}), 7.45(1 \mathrm{H}, \mathrm{t}, J=6.8 \mathrm{~Hz})$, $7.34(1 \mathrm{H}, \mathrm{d}, J=8.0 \mathrm{~Hz}), 7.26-7.29(1 \mathrm{H}, \mathrm{m}), 6.86(1 \mathrm{H}$, s), $4.91(2 \mathrm{H}, \mathrm{s}), 3.78(3 \mathrm{H}, \mathrm{s}), 2.62(1 \mathrm{H}, \mathrm{s}) ;{ }^{13} \mathrm{C} \mathrm{NMR}$ (100 MHz, $\left.\mathrm{CDCl}_{3}, \mathrm{TMS}\right) \delta: 189.2,159.4,145.7,141.8$, $125.9,123.2,121.5,120.5,120.2,118.9,117.6,108.8$, 92.1 (Aromatic C); 77.9, 76.6, 56.9, 29.4 (Aliphatic C); HRMS (ESI-MS) calcd. for $\mathrm{C}_{17} \mathrm{H}_{13} \mathrm{NO}_{2}(\mathrm{M}+\mathrm{H})$; 264.1024, found: 264.1024

\subsection{General procedure for the synthesis of dihydroquinalopyran [2,3-b]carbazoles $(5 a-f)$}

In a round bottom flask equipped with a magnetic stirring bar, $1.0 \mathrm{mmol}$ of aniline and $1.0 \mathrm{mmol}$ of $O$-propargyl salicylaldehyde in $5 \mathrm{~mL}$ of ionic liquid $[\mathrm{Bmim}]\left[\mathrm{BF}_{4}\right]$ as solvent, was added $10 \mathrm{~mol} \%$ of $\mathrm{CuI}$. Reaction mixture was stirred at $90-95^{\circ} \mathrm{C}$. After completion of the reaction, as indicated by the TLC, water 
(20 $\mathrm{mL}$ ) was added to the crude reaction mass. Then aqueous layer was extracted with dichloromethane $(3 \times$ $20 \mathrm{~mL}$ ). The combined organic layers were dried over anhydrous $\mathrm{Na}_{2} \mathrm{SO}_{4}$, filtered and concentrated under the reduced pressure. Product was purified by column chromatography on silica gel (eluent: hexanes/ethyl acetate).

2.2a 9-Methyl-6,9-dihydroquinolino[2',3':4,5]pyrano [2,3-b]carbazole (5a): m.p. $198-200^{\circ} \mathrm{C}$; IR (KBr): 3024, 2841, 1174, 1059, $634 \mathrm{~cm}^{-1}$; ${ }^{1} \mathrm{H}$ NMR $(500 \mathrm{MHz}$, $\left.\mathrm{CDCl}_{3}, \mathrm{TMS}\right) \delta: 9.21(1 \mathrm{H}, \mathrm{s}), 8.21(2 \mathrm{H}, \mathrm{d}, J=8.0 \mathrm{~Hz})$, $7.88(1 \mathrm{H}, \mathrm{s}), 7.78(1 \mathrm{H}, \mathrm{d}, J=8.0 \mathrm{~Hz}), 7.31(1 \mathrm{H}, \mathrm{dt}$, $J 1=1.5 \mathrm{~Hz}$ and $J 2=7.0 \mathrm{~Hz}), 7.46-7.52(2 \mathrm{H}, \mathrm{m})$, $7.38(1 \mathrm{H}, \mathrm{d}, J=8.0 \mathrm{~Hz}), 7.30(1 \mathrm{H}, \mathrm{d}, J=7.5 \mathrm{~Hz})$, $6.99(1 \mathrm{H}, \mathrm{s}), 5.41(2 \mathrm{H}, \mathrm{s}), 3.82(3 \mathrm{H}, \mathrm{s}) ;{ }^{13} \mathrm{C} \mathrm{NMR}$ $\left(125 \mathrm{MHz}, \mathrm{CDCl}_{3}\right.$, TMS) $\delta: 156.9,150.4,148.6,144.0$, $141.9,130.7,129.5,129.1,127.4,127.2,125.7,125.3$, 125.2, 123.5, 120.4, 119.7, 119.2, 117.8, 116.0, 108.4, 96.0 (Aromatic C); 68.9, 29.2 (Aliphatic C); HRMS (ESI-MS) calcd. for $\mathrm{C}_{23} \mathrm{H}_{16} \mathrm{~N}_{2} \mathrm{O}(\mathrm{M}+\mathrm{H}) ; 337.1341$, found: 337.1344 .

2.2b 3,9-Dimethyl-6,9-dihydroquinolino [2',3':4,5] pyrano[2,3-b]carbazole (5b): m.p. $254-256^{\circ} \mathrm{C}$; IR (KBr): 3187, 2829, 1148, 1025, $659 \mathrm{~cm}^{-1} ;{ }^{1} \mathrm{H}$ NMR (400 MHz, $\left.\mathrm{CDCl}_{3}, \mathrm{TMS}\right) \delta: 9.16(1 \mathrm{H}, \mathrm{s}), 8.19(1 \mathrm{H}, \mathrm{d}$, $J=8.0 \mathrm{~Hz}), 8.09(1 \mathrm{H}, \mathrm{d}, J=8.4 \mathrm{~Hz}), 7.76(1 \mathrm{H}, \mathrm{s})$, $7.53-7.57(2 \mathrm{H}, \mathrm{m}), 7.47(1 \mathrm{H}, \mathrm{t}, J=7.6 \mathrm{~Hz}), 7.37(1 \mathrm{H}$, $\mathrm{d}, J=8.0 \mathrm{~Hz}), 7.27-7.31(1 \mathrm{H}, \mathrm{m}), 6.97(1 \mathrm{H}, \mathrm{s}), 5.38$ $(2 \mathrm{H}, \mathrm{s}), 3.80(3 \mathrm{H}, \mathrm{s}), 2.56(3 \mathrm{H}, \mathrm{s}) ;{ }^{13} \mathrm{C} \mathrm{NMR}(100 \mathrm{MHz}$, $\left.\mathrm{CDCl}_{3}, \mathrm{TMS}\right) \delta$ : 156.7, 149.6, 147.1, 143.8, 141.8, $135.5,131.8,130.2,128.7,127.2,126.4,125.3,125.2$, 123.5, 120.4, 119.6, 119.0, 117.6, 116.1, 108.4, 96.1 (Aromatic C); 69.0, 29.3, 21.6 (Aliphatic C); HRMS (ESI-MS) calcd. for $\mathrm{C}_{24} \mathrm{H}_{18} \mathrm{~N}_{2} \mathrm{O}(\mathrm{M}+\mathrm{H}) ; 351.1497$, found: 351.1499 .

2.2c 3-Methoxy-9-methyl-6,9-dihydroquinolino[2',3': 4,5]pyrano[2,3-b]carbazole (5c): m.p. $228-230^{\circ} \mathrm{C}$; IR (KBr): 3149, 2837, 1249, 1164, $618 \mathrm{~cm}^{-1}$; ${ }^{1} \mathrm{H}$ NMR (400 MHz, $\mathrm{CDCl}_{3}$, TMS) $\delta: 9.09(1 \mathrm{H}, \mathrm{s}), 8.17(1 \mathrm{H}, \mathrm{d}$, $J=7.6 \mathrm{~Hz}), 8.08(1 \mathrm{H}, \mathrm{d}), J=9.2 \mathrm{~Hz}), 7.72(1 \mathrm{H}, \mathrm{s})$, $7.44(1 \mathrm{H}, \mathrm{d}, J=7.2 \mathrm{~Hz}), 7.34-7.38(2 \mathrm{H}, \mathrm{m}), 7.25-7.28$ $(1 \mathrm{H}, \mathrm{m}), 7.01(1 \mathrm{H}, \mathrm{d}, J=2.4), 6.93(1 \mathrm{H}, \mathrm{s}), 5.36(2 \mathrm{H}$, s), $3.93(3 \mathrm{H}, \mathrm{s}), 3.77(3 \mathrm{H}, \mathrm{s}) ;{ }^{13} \mathrm{C} \mathrm{NMR}(100 \mathrm{MHz}$, $\left.\mathrm{CDCl}_{3}, \mathrm{TMS}\right) \delta: 157.3,156.5,148.1,144.5,143.6$, $141.8,130.5,129.6,128.1,125.5,125.2,123.5,121.8$, 120.4, 119.6, 119.0, 117.3, 116.1, 108.3, 105.4, 96.0 (Aromatic C); 68.9, 55.5, 29.2 (Aliphatic C); HRMS (ESI-MS) calcd. for $\mathrm{C}_{24} \mathrm{H}_{18} \mathrm{~N}_{2} \mathrm{O}_{2}(\mathrm{M}+\mathrm{H}) ; 367.1446$, found: 367.1451 . 2.2d 3-Fluoro-9-methyl-6,9-dihydroquinolino[2',3': 4,5]pyrano[2,3-b]carbazole (5d): m.p. $241-243^{\circ} \mathrm{C}$; IR (KBr): $3125,2835,1451,1179,632 \mathrm{~cm}^{-1} ;{ }^{1} \mathrm{H}$ NMR $\left(500 \mathrm{MHz}, \mathrm{CDCl}_{3}, \mathrm{TMS}\right) \delta: 9.08(1 \mathrm{H}, \mathrm{s}), 8.16-8.18$ $(2 \mathrm{H}, \mathrm{m}), 7.69(1 \mathrm{H}, \mathrm{s}), 7.45-7.47(2 \mathrm{H}, \mathrm{m}), 7.28-7.35$ $(3 \mathrm{H}, \mathrm{m}), 6.91(1 \mathrm{H}, \mathrm{s}), 5.34(2 \mathrm{H}, \mathrm{s}), 3.74(3 \mathrm{H}, \mathrm{s}) ;{ }^{13} \mathrm{C}$ NMR (125 MHz, $\mathrm{CDCl}_{3}$, TMS) $\delta: 161.0,159.0,156.7$, $149.8,145.5,143.9,141.8,131.4,131.3,129.91,129.9$, 127.7, 127.6, 126.0, 125.3, 123.4, 120.7, 120.3, 119.7, $119.5,119.3,119.1,117.5,115.7,110.6,110.5,108.8$, 108.4, 96.0, 94.9 (Aromatic C); 68.7, 29.1 (Aliphatic C); HRMS (ESI-MS) calcd. for $\mathrm{C}_{23} \mathrm{H}_{15} \mathrm{FN}_{2} \mathrm{O}(\mathrm{M}+\mathrm{H})$; 355.1246, found: 355.1246 .

2.2e 3-Chloro-9-methyl-6,9-dihydroquinolino[2',3': 4,5]pyrano[2,3-b]carbazole (5e): m.p. $212-214^{\circ} \mathrm{C}$; IR (KBr): 3032, 2857, 1364, 1157, $629 \mathrm{~cm}^{-1} ;{ }^{1} \mathrm{H}$ NMR $\left(500 \mathrm{MHz}, \mathrm{DMSO}-d_{6}\right.$, TMS $) \delta: 9.05(1 \mathrm{H}, \mathrm{s}), 8.23(1 \mathrm{H}$, $\mathrm{d}, J=7.5 \mathrm{~Hz}), 8.19(1 \mathrm{H}, \mathrm{s}), 8.15(1 \mathrm{H}, \mathrm{s}), 8.01(1 \mathrm{H}, \mathrm{d}$, $J=8.5 \mathrm{~Hz}), 7.85(1 \mathrm{H}, \mathrm{d}, J=8.5 \mathrm{~Hz}), 7.56(1 \mathrm{H}, \mathrm{d}$, $J=8.0 \mathrm{~Hz}), 7.46(1 \mathrm{H}, \mathrm{t}, J=7.0 \mathrm{~Hz}), 7.21-7.26(2 \mathrm{H}$, $\mathrm{m}), 5.45(2 \mathrm{H}, \mathrm{s}), 3.84(3 \mathrm{H}, \mathrm{s}) ;{ }^{13} \mathrm{C}$ NMR $(125 \mathrm{MHz}$, $\left.\mathrm{CDCl}_{3}, \mathrm{TMS}\right) \delta: 157.1,150.6,146.9,144.3,142.1$, $133.1,131.1,130.9,130.2,128.7,126.7,126.0,123.0$, $120.5,120.1,118.9,118.6,117.6,115.6,109.7,97.2$ (Aromatic C); 68.4, 29.7 (Aliphatic C); HRMS (ESIMS) calcd. for $\mathrm{C}_{23} \mathrm{H}_{15} \mathrm{ClN}_{2} \mathrm{O}(\mathrm{M}+\mathrm{H}) ; 371.0951$, found: 371.0953 .

$2.2 \mathrm{f}$ 3-Bromo-9-methyl-6,9-dihydroquinolino $\left[2^{\prime}, 3^{\prime}\right.$ : 4,5]pyrano[2,3-b]carbazole (5f): m.p. $223-225^{\circ} \mathrm{C}$; IR (KBr): 3048, 2874, 1483, 1148, $625 \mathrm{~cm}^{-1}$; ${ }^{1} \mathrm{H}$ NMR (400 MHz, $\left.\mathrm{CDCl}_{3}, \mathrm{TMS}\right) \delta: 9.06(1 \mathrm{H}, \mathrm{s}), 8.15(1 \mathrm{H}, \mathrm{d}$, $J=7.2 \mathrm{~Hz}), 8.00(1 \mathrm{H}, \mathrm{d}, J=8.8 \mathrm{~Hz}), 7.82(1 \mathrm{H}, \mathrm{s})$, $7.74(1 \mathrm{H}, \mathrm{dd}, J 1=7.2 \mathrm{~Hz}, J 2=2.0 \mathrm{~Hz}), 7.65(1 \mathrm{H}$, s), $7.46(1 \mathrm{H}, \mathrm{t}, J=7.6 \mathrm{~Hz}), 7.34(1 \mathrm{H}, \mathrm{d}, J=7.6 \mathrm{~Hz})$, 7.26-7.29 (1H, m), $6.89(1 \mathrm{H}, \mathrm{s}), 5.35(2 \mathrm{H}, \mathrm{s}), 3.74(3 \mathrm{H}$, $\mathrm{s}) ;{ }^{13} \mathrm{C} \mathrm{NMR}\left(100 \mathrm{MHz}, \mathrm{CDCl}_{3}, \mathrm{TMS}\right) \delta: 156.7,150.7$, 147.0, 144.0, 141.8, 132.8, 130.6, 129.6, 129.4, 128.2, 126.1, 125.4, 123.4, 120.4, 119.7, 119.2, 119.1, 117.7, 115.5, 108.4, 96.0 (Aromatic C); 68.7, 29.2 (Aliphatic C); HRMS (ESI-MS) calcd. for $\mathrm{C}_{23} \mathrm{H}_{15} \mathrm{BrN}_{2} \mathrm{O}(\mathrm{M}+\mathrm{H})$; 415.0446, found: 415.0446 .

\section{Results and Discussion}

We choose 2-hydroxy-3-formylcarbazole (1) which would be a potential starting material for making pyrano[2,3-b]carbazole derivatives. The synthesis of $O$-propargylaldehyde precursor $\mathbf{3}$ is shown in scheme 1 . Demethylation of 2-methoxy-3-formylcarbazole ${ }^{8}$ (1) 


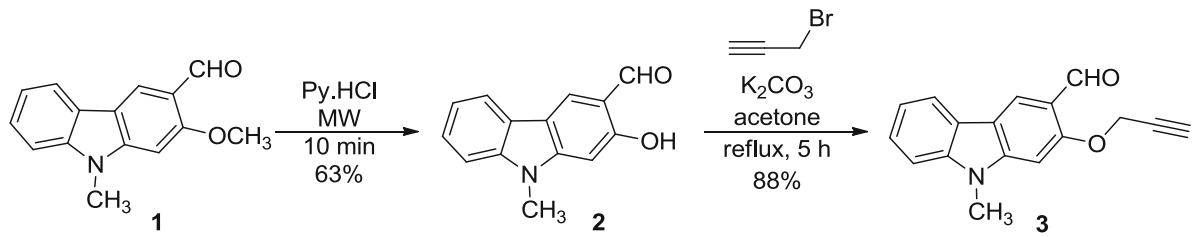

Scheme 1. Synthesis of 9-methyl-2-(prop-2-yn-1-yloxy)-9H-carbazole-3-carbaldehyde (3).

Table 1. Optimization of the reaction conditions.

\begin{tabular}{lccc} 
& & \\
\hline
\end{tabular}

General condition: aldehyde (1) $(1 \mathrm{mmol})$, aniline (2a) $(1.2 \mathrm{mmol})$ and $\mathrm{CuI}$ (10 mol\%). For entries 1,2 and 4,5 reflux temperature of corresponding solvents were maintained. For entries 3, 6 and 7 the reaction temperature $90-95^{\circ} \mathrm{C}$ were maintained.

${ }^{\text {a }}$ Yield of isolated product.

${ }^{\mathrm{b}}$ Without CuI.

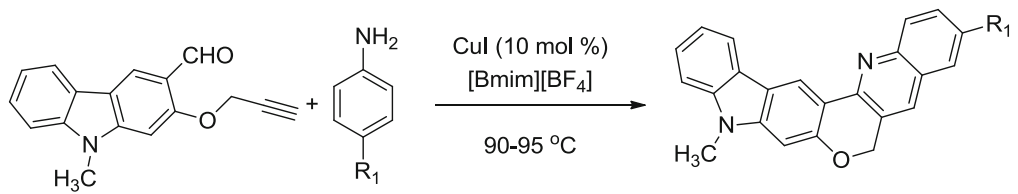<smiles>Cn1c2ccccc2c2cc3c(cc21)COc1cc2ccccc2nc1-3</smiles>

5a, 2.5 h, $78 \%$

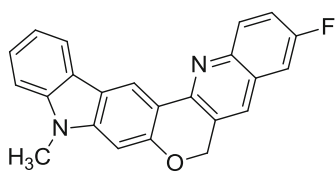

$5 d, 5$ h, $76 \%$

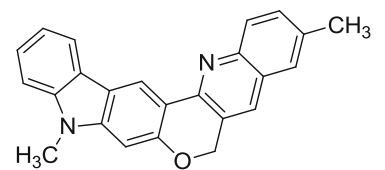

5b, 2 h, $81 \%$

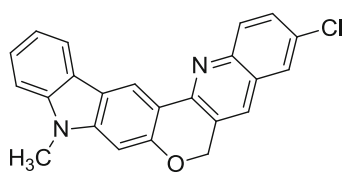

$5 e, 4.5 \mathrm{~h}, 75 \%$

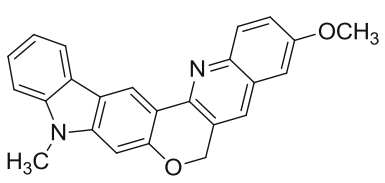

$5 c, 2$ h, $83 \%$

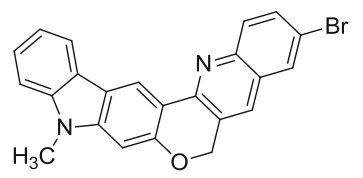

$5 f, 5$ h, $72 \%$

Scheme 2. Synthesis of pyranocarbazole derivatives (5a-f). 


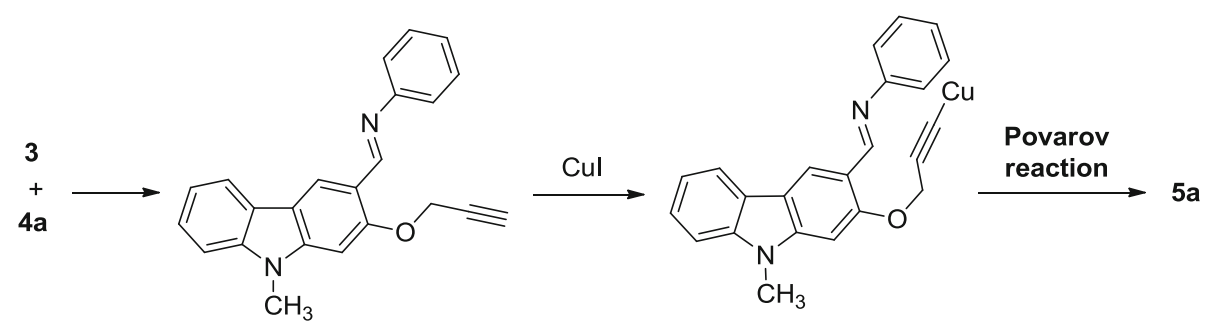

Scheme 3. Proposed mechanism for the Povarov reaction.

using pyridine hydrochloride salt under microwave condition afforded 2-hydroxy-3-formylcarbazole (2). $O$ Propargylation of compound $\mathbf{2}$ provided propargylated 3-formylcarbazole 3 in quantitative yield using propargyl bromide/acetone under reflux condition.

Then, we carried out the cyclization reaction of $O$ propargyl derivative $\mathbf{3}$ with aniline $\mathbf{4 a}$ in the presence of $\mathrm{CuI}$ as it showed better catalytic activity in the synthesis of chrominoquinoline and pyrroloquinoline derivatives. ${ }^{6 \mathrm{e}-\mathrm{g}} \mathrm{We}$ screened various solvents such as $\mathrm{CH}_{3} \mathrm{CN}$, toluene, THF, dioxane, DMSO and [Bmim] $\left[\mathrm{BF}_{4}\right]$ (table 1, entries 1-7). We found that the conditions using $[\mathrm{Bmim}]\left[\mathrm{BF}_{4}\right]$ as solvent with $10 \mathrm{~mol} \%$ of $\mathrm{CuI}$ at $90^{\circ} \mathrm{C}$ gave comparatively good yield (entry 6). Notably, without $\mathrm{CuI}$ in the presence of ionic liquid $[\mathrm{Bmim}]\left[\mathrm{BF}_{4}\right]$, the yield decreased.

With acceptable conditions in hand (table 1, entry 6), we tested the scope of this methodology for different aromatic amines (scheme 2). As shown in scheme 2, electron donating (-Me, -OMe) as well as electron withdrawing $(-\mathrm{F},-\mathrm{Cl},-\mathrm{Br})$ groups containing anilines worked well in the present system to give quinolinopyranocarbazole derivatives in good yields. The proposed reaction mechanism is shown in scheme 3 .

\section{Conclusions}

Copper catalysed Povarov reaction to synthesize quinolinopyrano[2,3- $b]$ carbazole derivatives has been developed. Applying various aromatic amines with $O$ propargylated carbazole aldehyde in the presence of $\mathrm{CuI}$ and $[\mathrm{Bmim}]\left[\mathrm{BF}_{4}\right]$ afford the corresponding pyranocarbazole products in a straightforward manner. This simple, efficient, one-pot and high yielding procedure would be a useful synthetic tool for the synthesis of pyranocarbazoles.

\section{Supplementary Information}

${ }^{1} \mathrm{H},{ }^{13} \mathrm{C}$ spectra and HRMS of compounds $\mathbf{2 , 3}$ and $\mathbf{5 a}-\mathbf{f}$ are mentioned in the supporting information. The electronic supporting information can be seen at www. ias.ac.in/chemsci.

\section{Acknowledgements}

We thank the Department of Science and Technology (DST) for financial assistance (Project number: SR/ S1/OC-70/2008). SR thanks the Council of Scientific and Industrial Research (CSIR) for Senior Research Fellowship.

\section{References}

1. (a) Schmidt A W, Reddy K R and Knölker H-J 2012 Chem. Rev. 112 3193; (b) Cordell G A 2008 The Alkaloids 65; (c) Knölker H-J 2008 The Alkaloids (Cordell G A ed.) Academic Press, Amsterdam 65 1; (d) Knölker H-J and Reddy K R 2002 Chem. Rev. 102 4303; (e) Gribble G W 1990 The Alkaloids; Brossi A (ed.) (San Diego, CA: Academic Press) p 239

2. (a) Jana A and Mal D 2010 Chem. Commun. 46 4411; (b) Lebold T P and Kerr M A 2008 Org. Lett. 10 997; (c) Mal D, Senapati B K and Pahari P 2007 Tetrahedron 63 3768; (d) Forke R, Krahl M P, Krause T, Schlechtingen G and Knölker H-J 2007 Synlett. 268; (e) Czerwonka R, Reddy K R, Baum E and Knölker H -J 2006 Chem. Commun. 711; (f) Knölker H-J, Fröhner W and Wagner A 1998 Tetrahedron Lett. 39 2947; (g) Krahl M P, Jager A, Krause T and Knölker H -J 2006 Org. Biomol. Chem. 4 3215; (h) Knölker H -J and Wolfgang F 1997 Synlett. 1108

3. (a) Ramsewak R S, Nair M G, Strasburg G M, DeWitt D L and Nitiss J L 1999 J. Agric. Food Chem. 47 444; (b) Wang Y S, He H P, Shen M Y, Hong X and Hao X J 2003 J. Nat. Prod. 66 416; (c) Itoigawa M, Kashiwada Y, Ito $\mathrm{C}$, Furukawa H, Tachibana Y, Bastow K F and Lee K-H 2000 J. Nat. Prod. 63893

4. (a) Crombie L and Ponsford R 1968 Chem. Commun. 894; (b) Chakraborty D P, Chatterji D and Ganguli S N 1969 Chem. Ind. (London) 1662; (c) Gruner K K and Knölker H -J 2008 Org. Biomol. Chem. 6 3902; (d) Gruner K K, Hopfmann T, Matsumoto K, Jäger A, Katsuki T and Knölker H-J 2011 Org. Biomol. Chem. 9 2057; (e) Lebold T P and Kerr M A 2007 Org. Lett. 9 1883; (f) Talluri K C and Nagarajan R 2012 J. Chem. Sci. 124487 
5. (a) Shaheen F, Ahmad M, Khan S N, Hussain S S, Anjum S, Tashkhodjaev B, Turgunov K, Sultankhodzhaev M N, Choudhary M I and Rahman A-U 2006 Eur. J. Org. Chem. 10 2371; (b) Nikolai M E, Artem S K, Andrey A Y, Mikhail Y A, Igor V M and Alexander K 2007 J. Org. Chem. 72 3443; (c) María E, Benito R, Paula G, Juan R, Carlos C, Humberto C and Jesus A 2010 Fitoterapia 81 66; (d) Zhi L, Tegley C M, Pio B, Edward J P, Motamedi M, Jones T D, Marschke K B, Mais D E, Risek B and Schrader W T 2003 J. Med. Chem. 46 4104; (e) Coghlan M J, Kym P R, Elmore S W, Wang A X, Luly J R, Wilcox D, Stashko M, Lin C W, Miner J, Tyree C, Nakane M, Jacobson P and Lane B C 2001 J. Med. Chem. 44 2879; (f) Ku Y Y, Grieme T, Raje P, Sharma P, Morton H E, Rozema M and King S A 2003 J. Org. Chem. 683238

6. (a) Tomashevskaya $\mathrm{M} \mathrm{M}$, Tomashenko $\mathrm{O} \mathrm{A}$, Tomashevskii A A, Sokolov V V and Potekhin A A 2007
Russ. J. Org. Chem. 43 77; (b) Sabitha G, Babu R S, Reddy B V S and Yadav J S 1999 Synth. Commun. 29 4403; (c) Rabin B, Dhananjaya S N S, Ramu B, Sai U K, Rajender K, Mukkanti K and Pal M 2008 Tetrahedron 64 582; (d) Vu A T, Campbell A N, Harris H A, Unwalla R J, Manas E S and Mewshaw R E 2007 Bioorg. Med. Chem. Lett. 17 4053; (e) Ramesh S, Gaddam V and Nagarajan R 2010 Synlett. 757; (f) Gaddam V, Ramesh S and Nagarajan R 2010 Tetrahedron 66 4218; (g) Ramesh S and Nagarajan R 2011 Tetrahedron Lett. 524857

7. (a) Babu G and Perumal P T 2000 Aldrichimica Acta 33 16; (b) Kouznetsov V V 2009 Tetrahedron 65 2721; (c) Sridharan V, Suryavanshi P A and Menéndez J C 2011 Chem. Rev. 111 7157; (d) Povarov L S 1967 Russ. Chem. Rev. 36656

8. Díaz J L, Villacampa B, López-Calahorra, F and Velasco D 2002 Chem. Mater. 142240 